\title{
Placental erythropoietin expression is upregulated in growth-restricted fetuses with abnormal umbilical artery Doppler findings: a case-control study of monochorionic twins
}

\author{
Yao-Lung Chang', An-Shine Chao', Hsiu-Huei Peng' ${ }^{1}$ Shuenn-Dyh Chang' ${ }^{1}$ Kuan-Ju Chen', Po-Jen Cheng \\ and Tzu-Hao Wang ${ }^{1,2,3^{*}}$
}

\begin{abstract}
Background: We previously reported that fetal plasma erythropoietin (EPO) concentrations are significantly increased in growth-restricted fetuses with abnormal umbilical artery (UA) Doppler. During hypoxia in an ovine model, the primary site of fetal EPO synthesis was switched from the kidneys to the placenta. Therefore, we designed this study to evaluate human placental EPO gene expression and the correlation to fetal serum EPO concentration in growth-restricted fetuses in a monochorionic (MC) twin model.
\end{abstract}

Methods: In MC twin pairs, selective intrauterine growth restriction (SIUGR) was defined as the presence of (i) birth weight discordance of $>20 \%$ and (ii) a smaller twin with a birth weight less than the 10th percentile. Fetal UA and middle cerebral artery (MCA) Doppler were checked within 1 week before delivery. An abnormal UA Doppler was defined as persistently absent or reverse end-diastolic flow. Cerebroplacental ratio (CPR) was defined as MCApulsatility index (PI)/UA-PI. Fetal plasma EPO concentrations were measured in cord blood, and EPO gene expression was assayed in each twin's placental territory. The intertwin plasma EPO ratio was calculated as the cord plasma EPO level of the smaller (or sIUGR) twin divided by the EPO concentration of the larger (or appropriate-forgestational-age (AGA)) twin, and the intertwin placental EPO gene expression ratio was calculated similarly.

Results: Twenty-six MC twins were analyzed, including normal twins (Group 1, $n=9$ ), twins with sIUGR without UA Doppler abnormalities (Group 2,n=9), and twins with sIUGR and UA Doppler abnormalities (Group 3,n=8). The CPRs of smaller (sIUGR) fetuses were significantly decreased in Group 3 MC twins $(p<0.001)$, but not significantly different between Group 1 and Group 2. The highest fetal plasma EPO ratio and placental EPO gene expression ratio were identified in Group 3 MC twins $(p<0.001)$. The placental EPO gene expression ratios were significantly correlated with the fetal plasma EPO ratios (Pearson's correlation test, $p=0.004$ ).

Conclusion: This study provides evidence of increased placental EPO expression in MC twin fetuses with sIUGR and abnormal UA Doppler. Future studies are needed to confirm the similar role of placental EPO in severe IUGR singletons.

Keywords: Erythropoietin, Monochorionic twin, Intrauterine growth restriction, Placenta

\footnotetext{
* Correspondence: knoxtn@cgmh.org.tw

'Department of Obstetrics and Gynecology, Chang Gung Memorial Hospital,

Linkou, College of Medicine, Chang Gung University, No.5, Fu-Shin

RoadGwei-Shan District, Taoyuan City, Taiwan

${ }^{2}$ School of Traditional Chinese Medicine, College of Medicine, Chang Gung

University, No.5, Fu-Shin RoadGwei-Shan District, Taoyuan City, Taiwan

Full list of author information is available at the end of the article
}

(c) The Author(s). 2018 Open Access This article is distributed under the terms of the Creative Commons Attribution 4.0 International License (http://creativecommons.org/licenses/by/4.0/), which permits unrestricted use, distribution, and reproduction in any medium, provided you give appropriate credit to the original author(s) and the source, provide a link to the Creative Commons license, and indicate if changes were made. The Creative Commons Public Domain Dedication waiver (http://creativecommons.org/publicdomain/zero/1.0/) applies to the data made available in this article, unless otherwise stated. 


\section{Background}

In both fetuses and adults, erythropoietin (EPO) secretion is stimulated by low oxygen level [1]. In adults, EPO is produced predominantly by the kidneys. In human fetuses with congenital absence of bilateral kidneys [2] and animal fetuses with surgical nephrectomy [3, 4], plasma EPO and hematocrit concentrations remain normal, indicating that the fetal kidneys are not the only site for EPO production. In an ovine study of artificially induced maternal hypoxia, the primary site of fetal EPO synthesis was switched from the kidneys to the placenta [5]. Because EPO does not cross the placenta in humans [6], maternal EPO is not a source of EPO for the fetus.

MC twins share an identical genetic makeup and maternal physiology, but unequal placental sharing has been shown to result in the development of sIUGR $[7,8]$ . SIUGR, defined as the presence of IUGR in one twin while the other is appropriate for gestational age (AGA), occurs in $12 \%-15 \%$ of all MC twin pregnancies [7]. According to the UA Doppler findings of the IUGR twin [9], MC twins with selective IUGR were classified as three types: (1) normal with positive diastolic velocity, (2) persistent absent or reverse of end-diastolic velocity (AREDV) and (3) intermittent AREDV. When umbilical artery (UA) Doppler findings for sIUGR twins revealed persistent or intermittent UA-AREDF, the risk of intrauterine fetal demise was reported to increase [7, 9].

We have previously reported that fetal plasma EPO concentrations were significantly increased in monochorionic (MC) twins with selective intrauterine growth restriction (sIUGR) and umbilical artery Doppler abnormality [10]. Because the two fetuses in an MC twin pregnancy share an identical genetic makeup, the fetal plasma EPO concentrations of the AGA fetus can serve as an internal control for the corresponding levels detected in the sIUGR twin. In this setting, the detection of significant differences in fetal EPO concentrations in MC twin pregnancies with sIUGR and abnormal UA Doppler (accompanied by the lack of such differences in MC twin pregnancies without sIUGR) suggests changes in fetal plasma EPO might be associated with growth restriction and abnormal UA Doppler.

Since the contribution of the human placenta to fetal plasma EPO concentrations during hypoxia remains unclear, to fill this knowledge gap, we conducted this study to analyze the human placental EPO gene expression and correlate placental EPO expression ratios to fetal plasma EPO ratios in growth-restricted fetuses in a MC twin model.

\section{Methods}

This study was conducted from October 2014 to May 2016 at Chang Gung Memorial Hospital, Linkou Medical Center, Taiwan. The study protocol was approved by the Institutional Review Board of the Chang Gung
Medical Foundation (IRB \#101-4803A3). The inclusion criteria required that $\mathrm{MC}$ twins were delivered in our hospital, mothers with MC twins provided informed consent, and cord blood and placental tissues were successfully collected. The exclusion criteria were the presence of twin-to-twin transfusion syndrome (TTTS), monoamniotic twins, twin anemia-polycythemia sequence (TAPS), and congenital, structural or genetic malformations in the fetus. Because uterine contractions may modify the fetal plasma EPO levels [1], only MC twins delivered through cesarean section before the beginning of labor were included.

The diagnosis of TTTS was based on the Quintero criteria [11]. TAPS was diagnosed in the presence of a postnatal intertwin hemoglobin difference of $>8 \mathrm{~g} / \mathrm{dL}$ [12]. During the first trimester or early second trimester, $\mathrm{MC}$ twin pregnancies were identified using the following ultrasonographic criteria: (i) the presence of a single placenta, (ii) the presence of a thin dividing membrane, and (iii) the absence of a twin peak (lambda) sign. Monochorionicity was confirmed by obstetricians through postpartum examination of the placenta (presence of a single placenta with intertwin anastomoses).

\section{Measurements of pulsed-wave Doppler in umbilical artery (UA) and middle cerebral artery (MCA), and calculation of cerebroplacental ratio (CPR)}

Pulsed-wave Doppler UA and MCA studies were performed using GE Voluson 730 Expert ultrasound machines within seven days before delivery; if more than one examination data are available then the last examination data were collected. Measurements were obtained during fetal quiescence. Means of three consecutive automated measurements were recorded for analysis for each fetus at each examination. A mid-segment of a free loop of umbilical cord was chosen as the site of measurement. Persistent UA-AREDF was recognized as abnormal UA Doppler, whereas intermittent UA-AREDF was recognized as normal UA Doppler. Measurements of the MCA indices were performed in the proximal one third of the MCA at the vessel nearest the transducer with the angle of insonation close to $0^{\circ}$. CPR is defined as: MCA-pulsatility index (PI)/UA-PI.

In this study, sIUGR was also defined as the presence of (i) a birth weight discordance of $>20 \%$ and (ii) a smaller twin with a birth weight less than the 10th percentile $[13,14]$. Birth weight discordance was calculated as the difference between the fetal weights of the larger and smaller twins divided by the fetal weight of the larger twin, as follows: [(body weight of the AGA or larger twin - body weight of the sIUGR or smaller twin)/(body weight of the AGA or larger twin)] $\times 100 \%$. An abnormal UA Doppler was defined as persistent UA-AREDF. MC twins without sIUGR were defined as Group 1, those 
with sIUGR but without UA Doppler abnormalities as Group 2, and those with both sIUGR and UA Doppler abnormalities as Group 3.

\section{Collection of fetal blood}

After delivery of the fetus during cesarean section, the umbilical cord of each baby was doubly clamped. Before removing the placenta, surgeons on the operating table collected cord blood from the placenta by puncturing the umbilical vein. Cord blood was sent to the Department of Clinical Pathology for EPO measurements using a commercially available chemiluminescent immunometric assay (IMMULITE 2000 systems, Siemens, Los Angeles, USA). The intertwin EPO ratio was defined as the plasma EPO concentration of the sIUGR twin divided by the EPO concentration of the AGA twin.

\section{Inspection and collection of placental tissues}

Placentas were collected immediately after delivery. After the blood was drained from the umbilical vessels, the placenta was washed with ice-cold phosphate-buffered saline to remove all blood clots and was examined for the integrity of the cotyledons and membranes. The vascular equator was defined as a border drawn in the middle of the vascular zone on the chorionic fetal surface where the communicating vessels met. The placentas were cut along the vascular equator. We collected two to three pieces of fresh placental tissue $(0.5 \times 0.5 \times 0.5 \mathrm{~cm})$ from the placental territory of each MC twin. The placental biopsies were taken from the middle layer of the placenta between the maternal and fetal surfaces. Regions with obvious calcification or infarction were avoided. The placental specimens were briefly rinsed with normal saline to remove the blood. The placental tissues were kept in RNALater reagent (Thermo Fisher Scientific, Waltham, MA, USA) at $4{ }^{\circ} \mathrm{C}$ for 24-48 h. Subsequently, the tissues were transferred to new tubes and kept in a freezer at $-70{ }^{\circ} \mathrm{C}$ for long-term storage [15]. EPO gene expression was measured in each twin's placental territory.

\section{Placental EPO gene expression analyses}

Total RNA was isolated from placental tissues by using TRIzol reagent (Invitrogen), according to the manufacturer's instructions. Complementary DNA (cDNA) synthesis was performed as per the manufacturer's instructions by using the SuperScript III first-strand cDNA synthesis system (Invitrogen). Quantitative real-time (RT)-PCR was performed using the SYBR Green method (Invitrogen). Placental EPO expression was analyzed in duplicate and normalized to the GAPDH values by using the following primer sequences: EPO sense: 5'-caaggaggccgagaatatca-3, EPO antisense: 5'-tccatcctcttccaggcata-3', GAPDH sense: 5'-GGTA
TCGTGGAAGGACTCATGAC-3', GAPDH antisense: 5'-ATGCCAGTGAGCTTCCCGT-3'. RT-PCR data are presented as $\triangle \mathrm{Ct}$ (target gene expression normalized to the housekeeping gene) and the fold changes in expression [16]. The placental EPO gene expression ratio for the two placental territories in a twin pair is presented as the fold changes between the two fetuses in $\mathrm{MC}$ twins: (relative EPO expression levels of the smaller (sIUGR) twin)/ (relative EPO expression levels of the larger (AGA) twin).

\section{Data analysis and statistics}

Data were analyzed using SPSS 11.0 statistical software (SPSS Inc., Chicago, IL, USA). Intergroup comparisons of continuous variables among the three study groups were performed using one-way analysis of variance (ANOVA), followed by post hoc at least significance difference (LSD) tests for multiple comparisons. Paired Student's $t$ tests were used to compare continuous data within each pair. The correlations between the plasma EPO ratio and placental EPO gene expression ratio were analyzed using Pearson's correlation coefficients. Two-tailed $p$ values of $<0.05$ were considered statistically significant.

\section{Results}

Twenty-six MC twin pairs met the inclusion criteria and were selected for this study. They were classified as Group $1(n=9)$, normal MC twins; Group $2(n=9)$, MC twins with sIUGR but without UA Doppler abnormalities; and Group $3(n=8)$, MC twins with sIUGR and UA Doppler abnormalities.

There was no significant difference in maternal age at delivery among the three groups of MC twins. MC twins in Group 3 were delivered significantly earlier (mean: 30.7 weeks of gestation) than those in Groups 1 and 2 . The birth weights of both AGA and sIUGR twins were lowest in Group 3 and highest in Group 1; likely reflecting the shortest gestational age of delivery in Group 3 and longest in Group 1. The observed birth weight discordance was most severe in Group 3, mainly due to the lowest birth weights being observed in the sIUGR fetuses of this group. The maternal body mass indices (BMIs) were not significantly different among the three groups of MC twins. No significant difference was observed in the hemoglobin levels between the two fetuses in any of the groups of MC twins (Table 1).

The placental EPO gene expression ratios in Group 3 were significantly higher than those in Groups 1 and 2 MC twins, but no significant differences were noted between the twin pregnancies in Groups 1 and 2 (Fig. 1). The fetal plasma EPO ratios in Group 3 were significantly higher than those in Groups 1 and 2 (Table 1). Cerebroplacental ratios (CPRs) derived from MCA and UA pulsatility index (PI) were not significantly different 
Table 1 Characteristics of three groups of monochorionic twin pregnancies

\begin{tabular}{|c|c|c|c|c|}
\hline & Group $1(n=9)$ & Group $2(n=9)$ & Group $3(n=8)$ & $p$ value \\
\hline Maternal age at delivery & $31.4 \pm 6.2$ & $31.1 \pm 3.2$ & $32.4 \pm 2.2$ & $0.80^{\#}$ \\
\hline Gestational age of delivery (weeks) & $36.7 \pm 0.97$ & $34.8 \pm 1.7$ & $30.7 \pm 2.7$ & $<0.001^{\#}$ (Group1 > Group2 > Group 3) \\
\hline Birth weight of the AGA (larger) twin (g) & $2597 \pm 271$ & $2237 \pm 395$ & $1398 \pm 403$ & $<0.001^{\#}$ (Groups $1>$ Group $2>$ Group 3) \\
\hline Birth weight of the sIUGR (smaller) twin (g) & $2333 \pm 276$ & $1573 \pm 300$ & $717 \pm 232$ & $<0.001^{\#}$ (Groups $1>$ Group $2>$ Group 3) \\
\hline Birth weight discordance (\%) & $10.0 \pm 6.6$ & $29.5 \pm 7.4$ & $48.9 \pm 7.4$ & $<0.001^{\#}$ (Groups $3>$ Group $2>$ Group 1) \\
\hline Cord plasma EPO-ratio & $1.03 \pm 0.20$ & $1.36 \pm 0.76$ & $1.95 \pm 0.59$ & $0.01^{\#}($ Group3 > Groups 1 and 2) \\
\hline $\begin{array}{l}\text { Cord plasma EPO of larger (AGA) fetus } \\
(\mathrm{mlU} / \mathrm{mL})\end{array}$ & $14.2 \pm 11.4$ & $10.5 \pm 9.2$ & $24.7 \pm 36.8$ & $0.410^{\#}$ \\
\hline Cord plasma EPO of smaller (sIUGR) fetus (mIU/ mL) & $16.0 \pm 14.6$ & $12.7 \pm 9.3$ & $49.4 \pm 71.8$ & $0.153^{\#}$ \\
\hline Maternal BMI at delivery & $26.6 \pm 1.9$ & $27.4 \pm 3.4$ & $27.1 \pm 3.2$ & $0.880^{\#}$ \\
\hline CPR of larger (AGA) fetus & $1.39 \pm 0.21$ & $1.47 \pm 0.29$ & $1.74 \pm 0.29$ & $0.064^{\#}$ \\
\hline CPR of smaller (sIUGR) fetus & $1.41 \pm 0.20$ & $1.33 \pm 0.13$ & $0.45 \pm 0.03$ & $<0.001^{\#}$ (Group3 < Groups 1 and 2) \\
\hline Neonatal hemoglobin levels (larger/AGA) (g/dL) & $14.8 \pm 1.2$ & $16.4 \pm 1.8$ & $16.8 \pm 1.1$ & $※$ \\
\hline Neonatal hemoglobin levels (smaller/sIUGR) (g/dL) & $14.4 \pm 1.2$ & $16.5 \pm 2.3$ & $15.6 \pm 1.7$ & $※$ \\
\hline
\end{tabular}

Group 1: normal MC twins; group 2: twins with sIUGR without UA Doppler abnormalities; group 3: twins with sIUGR and UA Doppler abnormalities

Birth-weight discordance: [(body weight of the larger or AGA twin - body weight of the smaller or sIUGR twin) / body weight of the larger or AGA twin] $\times 100 \%$

Cord plasma EPO-ratio: [plasma EPO level of the smaller (or the small-for-gestational-age) twin] / [plasma EPO concentration of the larger (or the

appropriate-for-gestational-age) twin]

CPR (cerebroplacental ratio) $=$ (middle cerebral artery-pulsatility index) / (umbilical artery- pulsatility index)

Abbreviations: $M C$ monochorionic, sIUGR selective intrauterine growth restriction, $A G A$ appropriate-for-gestational-age, $E P O$ erythropoietin, $C P R$

cerebroplacental ratio

\#One-way analysis of variance

※o significant difference was observed in the hemoglobin levels between the two fetuses in any of the groups of MC twins

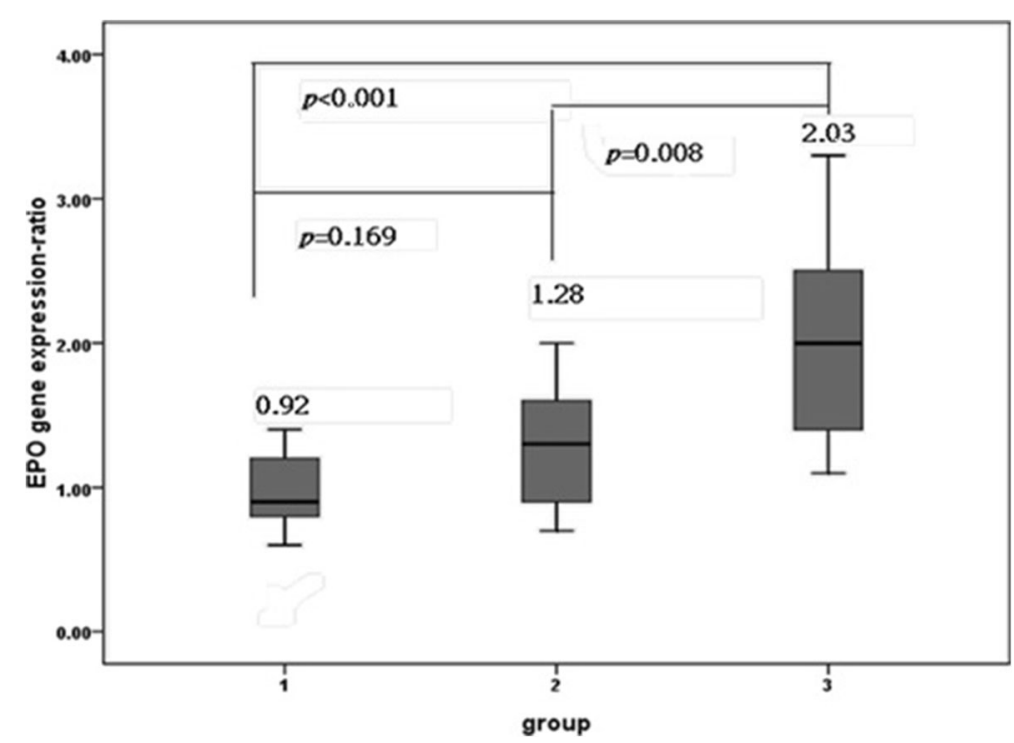

Fig. 1 Placental EPO gene expression ratios in three groups of $M C$ twins. The placental EPO gene expression ratio was significantly higher in Group 3 (one-way ANOVA, $p<0.001$ ), but no significant differences were observed between Groups 1 and 2 (post hoc comparison using the LSD test). The placental EPO gene expression ratios for the two placental territories in a twin pair are presented as fold changes between the two fetuses in MC twins: (the relative EPO expression concentrations of the sIUGR twin)/ (relative EPO expression concentrations of the larger twin). A higher EPO gene expression ratio (as shown in group 3) indicated more EPO expression by the placental territory of the sIUGR twin. Data are expressed as means and ranges of values. Group 1: normal MC twins; Group 2: twins with sIUGR but without UA Doppler abnormalities; and Group 3: twins with sIUGR and UA Doppler abnormalities. Abbreviations: EPO: erythropoietin; MC: monochorionic; sIUGR: selective intrauterine growth restriction; AGA: appropriate for gestational age; UA: umbilical artery; LSD: least significant difference 
among larger (AGA) fetuses in three groups of MC twins. However, CPRs of the smaller (sIUGR) fetuses were significantly decreased in groups $3 \mathrm{MC}$ twins, but not significantly different between group 1 and $2 \mathrm{MC}$ twins (Table 1). The placental EPO gene expression ratios were significantly correlated with the fetal plasma EPO ratios (Pearson's correlation test, $p=0.004$; Fig. 2).

\section{Discussion}

This study reports that the placental expression of EPO in human twin pairs is associated with fetal growth restriction and abnormal UA Doppler. We did not include TTTS and TAPS in this study, so we could examine individual placental gene expression without interference by communicating blood vessels between twins. Both placental territories of $\mathrm{MC}$ twins share an identical genetic makeup, but the sIUGR fetus with abnormal UA Doppler findings has a smaller placental territory and receives less placental perfusion [7, 8]. Therefore, we hypothesized that hypoperfusion to the severe sIUGR fetus may stimulate its placental EPO expression. Our current results (Table 1, Figs. 1 and 2) support this hypothesis.

Group $3 \mathrm{MC}$ twins were delivered earliest. Given the lack of significant correlations between the gestational age at delivery and plasma EPO concentrations in the sIUGR or AGA fetuses [10], we could hypothesize that the increased cord plasma EPO ratios and placental EPO gene expression ratios in Group $3 \mathrm{MC}$ twins may not be caused by early delivery. Notably, good correlations were observed between the cord plasma EPO ratios and placental EPO expression ratios (Fig. 2), strongly supporting our hypothesis that the upregulated placental EPO gene expression in Group $3 \mathrm{MC}$ twins accounts for the increased fetal plasma EPO concentrations in fetuses with hypoperfusion. The placenta has been long considered as a neuroendocrine organ $[17,18]$, and neuroepithelial cells have been shown to produce EPO in the earliest stage of life in mouse embryos [19]. These reports collectively support the notion that the placenta can be the site of EPO generation.

In addition to stimulating the production of red blood cells, EPO was recently shown to have functions such as inhibition of apoptosis and stimulation of inflammatory and proangiogenic mechanisms in multiple organ systems [20]. These nonerythroid functions of EPO have been considered to protect tissues from hypoxia and ischemia/reperfusion injury [21, 22]. The pleiotropic cytokine EPO was reported to significantly attenuate brain injury induced by repeated lipopolysaccharide (LPS) exposure [23]. Treatment with recombinant human EPO can also ameliorate LPS-induced damage to the placenta and fetal liver [24]. In this study, no significant differences were observed in the hemoglobin levels between the AGA and sIUGR fetuses in Group 3. Therefore, the increase in fetal plasma EPO concentrations is unlikely to only promote erythropoiesis; instead, it may have a role in tissue protection from decompensated hypoxia in sIUGR fetuses.

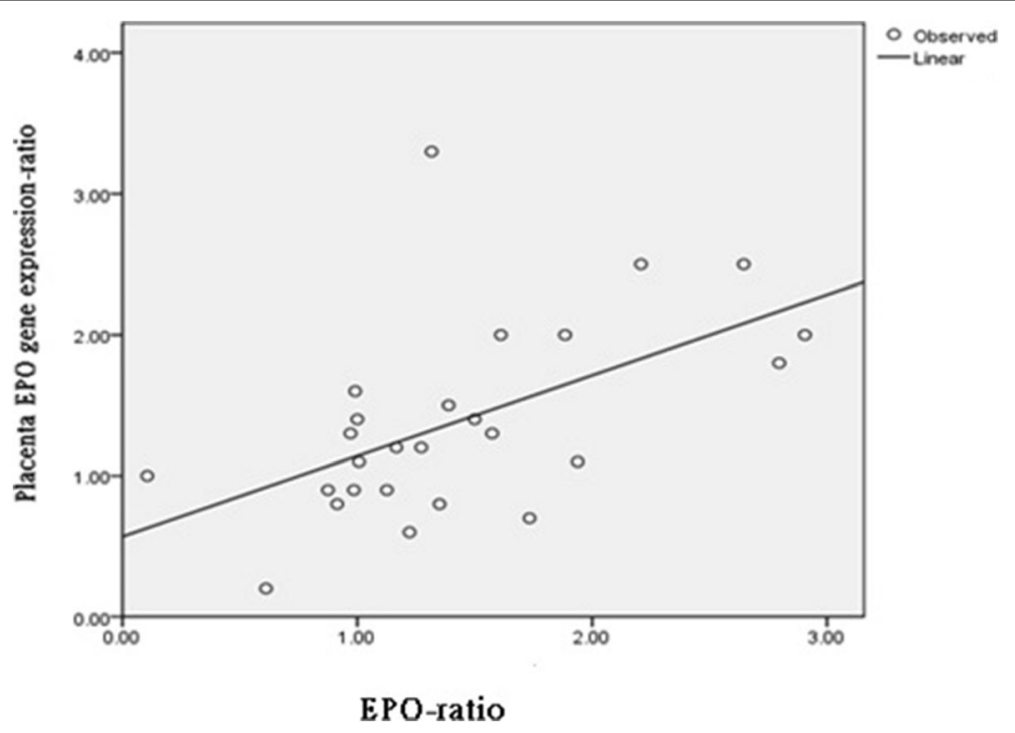

Fig. 2 Positive correlations between the cord plasma EPO ratios and placental EPO gene expression ratios. The cord plasma EPO ratio is plasma EPO concentration in the smaller (or the small-for-gestational-age) twin divided by the EPO concentration of the larger (or the appropriate-forgestational-age) twin. The placental EPO gene expression ratios for the two placental territories in a twin pair are presented as fold changes between the two fetuses in MC twins: (relative EPO expression concentrations of the sIUGR twin) / (relative EPO expression concentrations of the AGA twin). Abbreviations: EPO: erythropoietin; MC: monochorionic; sIUGR: selective intrauterine growth restriction; AGA: appropriate for gestational age 
In fetuses with chronic hypoxia, the UA-PI increase but MCA-PI decrease, reflecting a brain sparing process [25]. The ratio of MCA-PI to UA-PI, named as the cerebroplacental ratio (CPR), has been shown to be a better proxy marker for fetal compromise during placental insufficiency and hypoxemia. Discordant CPR is an independent predictor for the risk of perinatal loss in twin pregnancies [26]. In group $3 \mathrm{MC}$ twins, the sIUGR fetuses had the lowest CPRs, indicating that group 3 sIUGR fetuses had the severest insufficiency of placental perfusion. These results support that the higher placental EPO expression ratio and the higher fetal plasma EPO concentration ratio may be caused by malperfusion to the placental territory of the sIUGR fetus.

The use of MC twin models is instrumental in clearly identifying the increased EPO expression levels in the less perfused placenta, because an MC twin model provides identical genetic and environmental conditions, minimizing individual, basal noises in gene expression. Thus, analysis of MC twins can reveal subtle changes that are impossible to observe in other pregnant human case-control studies.

The limitations of this study are as follows. First, the histological site of EPO production in the human placenta could not be identified, although EPO mRNA has been detected at the apical tips of the fetal villi in the inner margin of the caruncle [27]. Second, we could not quantify the proportion, if not all, of the fetal plasma EPO amount derived from the placenta in the setting of uncompensated hypoxia. However, the placental EPO gene expression ratios were well correlated with the fetal plasma EPO ratios. Third, individual disease severity forced us to deliver twin babies at different ages of gestation, resulting in a significant difference in the gestational ages at delivery and birth weight of the AGA twins among the three groups. Despite our endeavors to minimize such confounding age factors by only comparing inter-twin differences in each pair of twins (Fig. 1), we still cannot completely rule out that differences in gestational age do not affect cord plasma EPO ratios at all. Finally, because many women in this study were referred by other hospitals, we lacked information regarding the pregestational BMI and maternal body weight gain during pregnancy. Therefore, we could not evaluate the potential involvement of placental lipotoxic environments induced by maternal obesity [28].

\section{Conclusion}

Results of this MC twin study provide human evidence of increased placentalEPO expression in sIUGR fetuses with abnormal UA Doppler. The observed increased EPO expression in this group is correlated with increased plasma EPO concentrations.

\section{Abbreviations}

AGA: Appropriate for gestational age; ANOVA: Analysis of variance; AREDF: Absent or reverse end-diastolic flow; CPR: Cerebroplacental ratio; EPO: Erythropoietin; LPS: Lipopolysaccharide; LSD: Least significance difference; MC: Monochorionic; MCA: Middle cerebral artery; PI: Pulsatility index; rhEPO: recombinant human erythropoietin; sIUGR: selective intrauterine growth restriction; TAPS: Twin anemia-polycythemia sequence; TITS: Twin-twin transfusion syndrome; UA: Umbilical artery

\section{Acknowledgments \\ We are grateful for the funding provided by Chang Gung Memorial Hospital,} Linkou Medical Center (CMRPG3D0183).

\section{Funding}

This work was supported by a grant (CMRPG3D0183) from Chang Gung Memorial Hospital, Linkou Medical Center. The role of the CMRPG3D0183 was to support the collections of specimens and laboratory procedures.

\section{Availability of data and materials}

The datasets obtained and/or analyzed during the current study are available from the corresponding author on reasonable request.

\section{Authors' contributions}

YLC and THW designed the study, performed laboratory experiments, collected and analyzed data, and wrote and revised the manuscript. YLC and ASC carried out all the clinical tasks and acquisition and interpretation of clinical data. HHP, SDC, KJC and PJC participated in clinical patient management, specimen collection, and acquisition and interpretation of data. All authors have contributed to drafting or revising the manuscript, approved this version to be published, and are willing to take public responsibility for the accuracy and integrity of its content.

\section{Ethics approval and consent to participate}

Institutional Review Board of the Chang Gung Medical Foundation (IRB \#1014803A3). Patients who provided written informed consents and had successfully donated cord blood and placental tissues were included in this study.

\section{Consent for publication}

Not applicable.

\section{Competing interests}

The authors declare that they have no competing interests.

\section{Publisher's Note}

Springer Nature remains neutral with regard to jurisdictional claims in published maps and institutional affiliations.

\section{Author details}

'Department of Obstetrics and Gynecology, Chang Gung Memorial Hospital, Linkou, College of Medicine, Chang Gung University, No.5, Fu-Shin RoadGwei-Shan District, Taoyuan City, Taiwan. ${ }^{2}$ School of Traditional Chinese Medicine, College of Medicine, Chang Gung University, No.5, Fu-Shin RoadGwei-Shan District, Taoyuan City, Taiwan. ${ }^{3}$ Genomic Medicine Research Core Laboratory (GMRCL), Chang Gung Memorial Hospital, No.5, Fu-Shin RoadGwei-Shan District, Taoyuan City, Taiwan.

Received: 22 September 2016 Accepted: 3 August 2018

Published online: 08 August 2018

References

1. Teramo KA, Widness JA. Increased fetal plasma and amniotic fluid erythropoietin concentrations: markers of intrauterine hypoxia. Neonatology. 2009;95(2):105-16.

2. Widness JA, Philipps AF, Clemons GK. Erythropoietin levels and erythropoiesis at birth in infants with potter syndrome. J Pediatr. 1990;117(1 Pt 1):155-8.

3. Lim GB, Moritz K, Jeyaseelan K, Wintour EM. Effect of hemorrhage and nephrectomy on erythropoietin gene expression in the ovine fetus. Mol Cell Endocrinol. 1996;117(1):101-9. 
4. Moritz KM, Macris M, Talbo G, Wintour EM. Foetal fluid balance and hormone status following nephrectomy in the foetal sheep. Clin Exp Pharmacol Physiol. 1999;26(11):857-64.

5. Davis LE, Widness JA, Brace RA. Renal and placental secretion of erythropoietin during anemia or hypoxia in the ovine fetus. Am J Obstet Gynecol. 2003;189(6):1764-70.

6. Widness JA, Schmidt RL, Sawyer ST. Erythropoietin transplacental passage-review of animal studies. J Perinat Med. 1995;23(1-2):61-70.

7. Chang YL, Chang SD, Chao AS, Hsieh PC, Wang CN, Wang TH. Clinical outcome and placental territory ratio of monochorionic twin pregnancies and selective intrauterine growth restriction with different types of umbilical artery Doppler. Prenat Diagn. 2009;29(3):253-6.

8. Chang YL, Chang SD, Chao AS, Wang CN, Wang TH, Cheng PJ. The relationships of umbilical venous volume flow, birthweight and placental share in monochorionic twin pregnancies with and without selective intrauterine growth restriction. Twin Res Hum Genet. 2011;14(2):192-7.

9. Gratacos E, Lewi L, Munoz B, Acosta-Rojas R, Hernandez-Andrade E, Martinez $J M$, Carreras E, Deprest J. A classification system for selective intrauterine growth restriction in monochorionic pregnancies according to umbilical artery Doppler flow in the smaller twin. Ultrasound Obstet Gynecol. 2007; 30(1):28-34.

10. Chang YL, Chao AS, Peng HH, Chang SD, Su SY, Chen KJ, Cheng PJ, Wang $\mathrm{TH}$. Increased fetal plasma erythropoietin in Monochorionic twin pregnancies with selective intrauterine growth restriction and abnormal umbilical artery Doppler. Twin Res Hum Genet. 2016;19(4):383-8.

11. Quintero RA, Morales WJ, Allen MH, Bornick PW, Johnson PK, Kruger M. Staging of twin-twin transfusion syndrome. J Perinatol. 1999;19(8 Pt 1):550-5.

12. Lopriore E, Slaghekke F, Oepkes D, Middeldorp JM, Vandenbussche FP, Walther FJ. Hematological characteristics in neonates with twin anemiapolycythemia sequence (TAPS). Prenat Diagn. 2010;30(3):251-5.

13. Russell Z, Quintero RA, Kontopoulos EV. Intrauterine growth restriction in monochorionic twins. Semin Fetal Neonatal Med. 2007;12(6):439-49.

14. Sueters $M$, Oepkes D. Diagnosis of twin-to-twin transfusion syndrome, selective fetal growth restriction, twin anaemia-polycythaemia sequence, and twin reversed arterial perfusion sequence. Best Pract Res Clin Obstet Gynaecol. 2014;28(2):215-26.

15. Wang TH, Lee YS, Chen ES, Kong WH, Chen LK, Hsueh DW, Wei ML, Wang HS, Lee YS. Establishment of cDNA microarray analysis at the genomic medicine research Core Laboratory (GMRCL) of Chang gung memorial hospital. Chang Gung Med J. 2004;27(4):243-60.

16. Chang SD, Chao AS, Peng HH, Chang YL, Wang CN, Cheng PJ, Lee YS, Chao A, Wang TH. Analyses of placental gene expression in pregnancy-related hypertensive disorders. Taiwan J Obstet Gynecol. 2011;50(3):283-91.

17. Toti P, Ciarmela P, Florio P, Volpi N, Occhini R, Petraglia F. Human placenta and fetal membranes express nerve growth factor mRNA and protein. J Endocrinol Investig. 2006;29(4):337-41.

18. Voltolini C, Petraglia F. Neuroendocrinology of pregnancy and parturition. Handb Clin Neurol. 2014;124:17-36.

19. Suzuki N. Erythropoietin gene expression: developmental-stage specificity, celltype specificity, and hypoxia inducibility. Tohoku J Exp Med. 2015;235(3):233-40.

20. van Patot MC, Ebensperger G, Gassmann M, Llanos AJ. The hypoxic placenta. High Alt Med Biol. 2012;13(3):176-84.

21. Mihov D, Bogdanov N, Grenacher B, Gassmann M, Zund G, Bogdanova A, Tavakoli R. Erythropoietin protects from reperfusion-induced myocardial injury by enhancing coronary endothelial nitric oxide production. Eur J Cardiothorac Surg. 2009;35(5):839-46. discussion 846

22. Pappo O, Ben-Ari Z, Shevtsov E, Avlas O, Gassmann M, Ravid A, Cheporko Y, Hochhauser $E$. The role of excessive versus acute administration of erythropoietin in attenuating hepatic ischemia-reperfusion injury. Can J Physiol Pharmacol. 2010;88(12):1130-7.

23. Rees $\mathrm{S}$, Hale N, De Matteo R, Cardamone L, Tolcos M, Loeliger M, Mackintosh A, Shields A, Probyn M, Greenwood D, et al. Erythropoietin is neuroprotective in a preterm ovine model of endotoxin-induced brain injury. J Neuropathol Exp Neurol. 2010;69(3):306-19.

24. Dijkstra F, Jozwiak M, De Matteo R, Duncan J, Hale N, Harding R, Rees S. Erythropoietin ameliorates damage to the placenta and fetal liver induced by exposure to lipopolysaccharide. Placenta. 2010;31(4):282-8.

25. Morales Rosello J, Hervas Marin D, Fillol Crespo M, Perales Marin A. Doppler changes in the vertebral, middle cerebral, and umbilical arteries in fetuses delivered after 34 weeks: relationship to severity of growth restriction. Prenat Diagn. 2012;32(10):960-7.
26. Khalil AA, Khan N, Bowe S, Familiari A, Papageorghiou A, Bhide A Thilaganathan B. Discordance in fetal biometry and Doppler are independent predictors of the risk of perinatal loss in twin pregnancies. Am J Obstet Gynecol. 2015;213(2):222 e221-10.

27. Kim MJ, Bogic L, Cheung CY, Brace RA. Expression of erythropoietin mRNA, protein and receptor in ovine fetal membranes. Placenta. 2001;22(10):846-51.

28. Saben J, Lindsey F, Zhong Y, Thakali K, Badger TM, Andres A, GomezAcevedo $\mathrm{H}$, Shankar K. Maternal obesity is associated with a lipotoxic placental environment. Placenta. 2014;35(3):171-7.

\section{Ready to submit your research? Choose BMC and benefit from:}

- fast, convenient online submission

- thorough peer review by experienced researchers in your field

- rapid publication on acceptance

- support for research data, including large and complex data types

- gold Open Access which fosters wider collaboration and increased citations

- maximum visibility for your research: over $100 \mathrm{M}$ website views per year

At BMC, research is always in progress.

Learn more biomedcentral.com/submissions 